This article has been scanned by iThenticat No plagiarism detected

Volume 3, Issue 2, April 2021

p. $98-108$

\title{
PEDAGOGICAL ENGINEERING FOR TEACHING ARABIC FOR EARLY CHILDHOOD -THE LEXICON AS A MODEL-
}

\author{
http://dx.doi.org/10.47832/2757-5403.2-3.9
}

\section{Fatima Az Zahra MONSEF1}

\begin{abstract}
:
The importance of the research project lies in the formation of the child during the pre-school stage by transforming him into the following mental stages in the development process, through the guiding function of the language, and the development of his logical thinking during the age stage between4-6 years, by enriching his linguistic outcome, and the formation of concepts, And the diversification of levels of linguistic knowledge within the environment to which the child belongs. The linguistic lexical project has educational and cultural goals, child's tongue, accompanied by some information related to their pronunciation, drawing, derivation with their synonyms, opposites, uses and employment.

Therefore, we discussed during this research project an explanation of the pedagogical background controlling the manufacture of this dictionary, from external characteristics at the level of from, and internal to the level of content, in addition to the principles, conditions and standards that should de, as follows: basic principles:

1. Simplicity

2. The functional dimension is words used in daily life that help the child to cope

3. Helping to continue the processes of mental development, sensory, motor, emotional.

4. Proximity to the child's surroundings and environment

A moong the conditions for compositing this dictionary is the development of contemporary scientific and artistic terms. To be characterized by clarity, comprehensiveness and arrangement, symbols, to be descriptive and analytical in the arrangement of its vocabulary, styles, and sentences.
\end{abstract}

Key words: Lexicon, Early Childhood, Arabic Language, Pedagogy.

\footnotetext{
${ }^{1}$ Dr. , Sidi Mohamed Ben Abdallah University, Morocco, fmonsef@fmps.ma, https://orcid.org/0000-0003$\underline{3263-238 X}$
}

Copyright (C) Published by IJHER Journal, www.ijherjournal.com Rimar Academy, Fatih, Istanbul, 34093 Turkey

All rights reserved 


\title{
الهندسة البيداغوجية لتعليم العربية للطقولة المبكرة ـالمعجم اللغوي أنموذجاً.
}

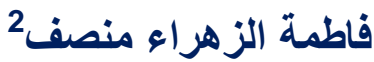

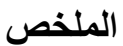

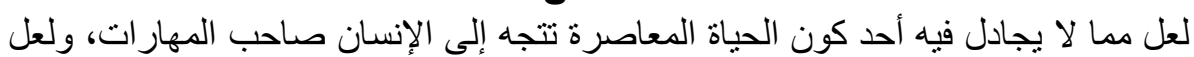

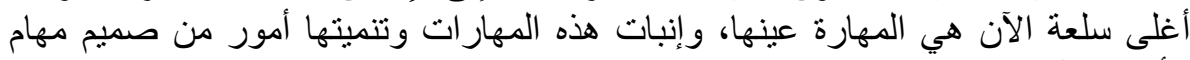

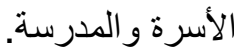
إذ يعتبر ضعف التحكم في اللغة المكتوبة و المنطوقة من قبل الطفل في المرحلة العمرية

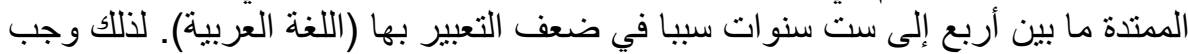

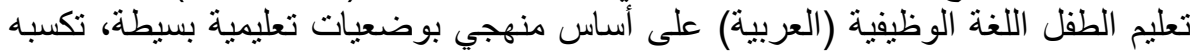

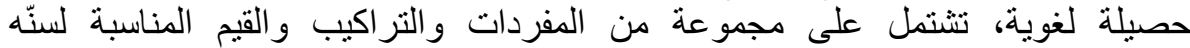

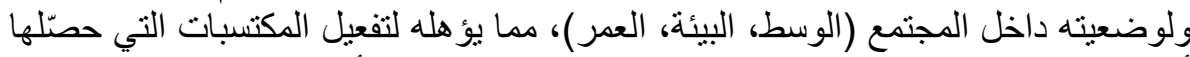

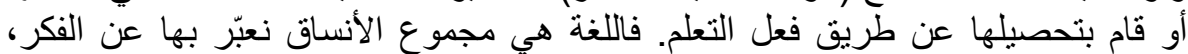

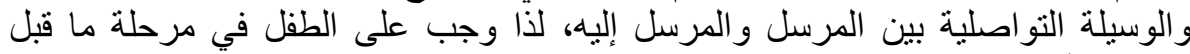

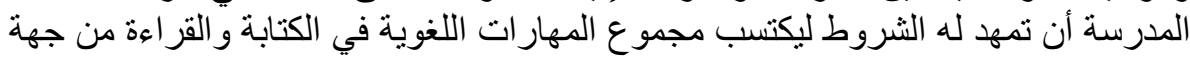

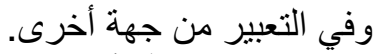
الكلمات المفتاحية: أصول التربية، اللغة العربية ، الطفولة المبكرة.
\end{abstract}

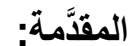

توفير البيئة اللغوية السليمة لتعليم الطفل يبدأ من إجراءات ومر احل أولية أساسية وهي إصلاح اللغة في بيئة ومناخ معين،

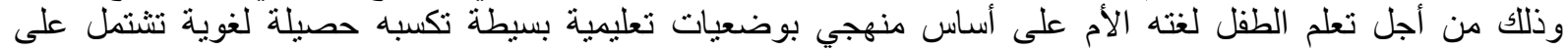

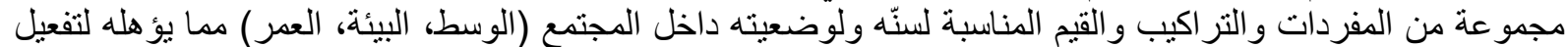

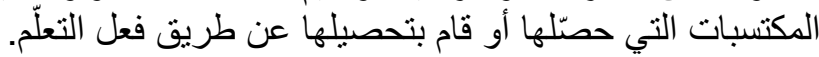

أهمية الاراسة: تكمن أهمية المشروع البحثي في تكوين الطفل في مرحلة ما قبل المدرسة عن طريق تحويله إلى المر احل الحبل

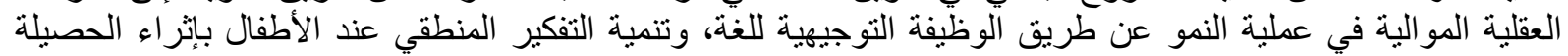

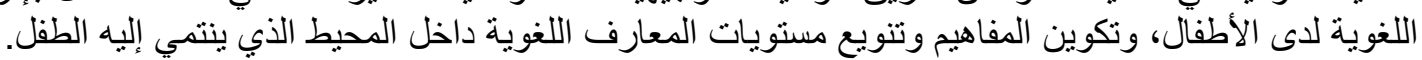

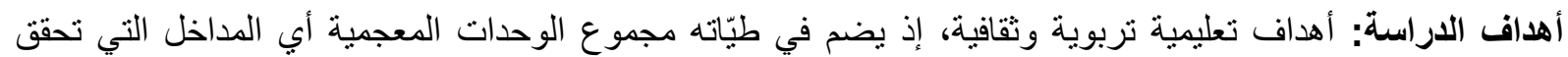

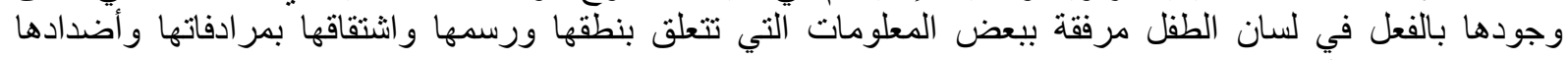

و واستعمالاتها وتوظيفها. 1. أن يجعل المتعلّم قادر ا على إدر الك علامات محيطه.

2. أن يجعل المعجم المتعلّم (الطفل) قادر النمل على استعمال علامات محيطه اللغوية( اللغة العربية)...استعمالا صحيحا مفيدا. 3. أن يساهم المعجم في بناء كفايات لدى المتعلّم، ويكون قادر ا من خلالها التعبير عن حاجاته.

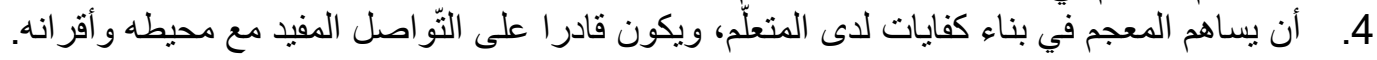

ربما هناك ضعف على مستولى الفهم والاستيعاب للمفردات والتراكيب اللغوية الخاصة باللغة العربية لدى الطفل،

$$
\text { فرضيات الاراسة: }
$$

$$
\text { نتيجة عدم قدرة الطفل على اكتساب المعارف و المهار الات اللغوية. }
$$




\section{PEDAGOGICAL ENGINEERING FOR TEACHING ARABIC FOR EARLY}

CHILDHOOD-THE LEXICON AS A MODEL-

ربما ما ينعكس على لغة الطفل نطقا وكتابة أثناء وبعد هذه المرحلة العمرية سببه عدم التنلائم في طر ائق تنمية

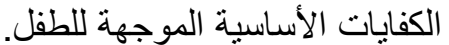

• ربما أن اللغة العربية التي يتعلمها الطفل غير وظيفية، لا تشتمل على مجموع المهارات والقواعد الضرورية

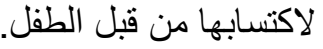

إثكالية الاراسة:

• أي مستوى من اللغة يمكن أن يعتمد في اختيار المادة اللغوية في تأليف المعجم ما قبل المدرسة؟

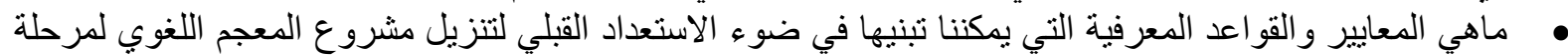

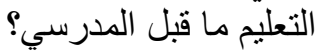
• ماهي أبرز المر احل والخطو ات؟ التي يمكننا قطعها من أجل تنزيل وتفعيل مخطط المشروع المعجي؟ المبحث الأول: المداخل والخلقيات - أولا: الخلفيات المهيكلة للمعجم اللغوي.

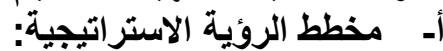

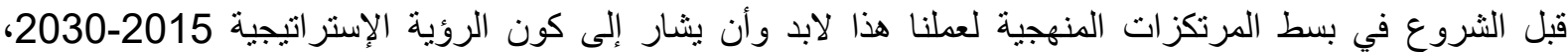

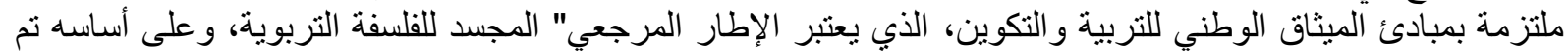

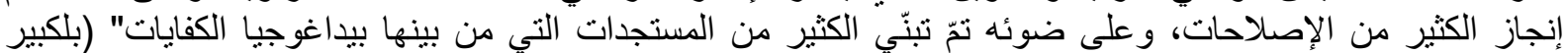

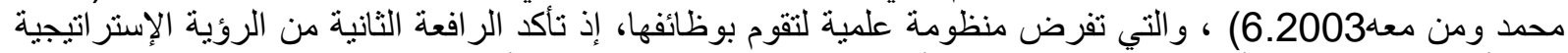

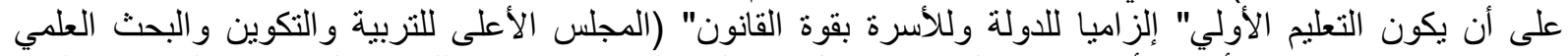

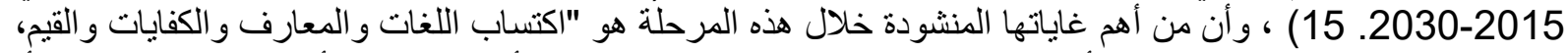

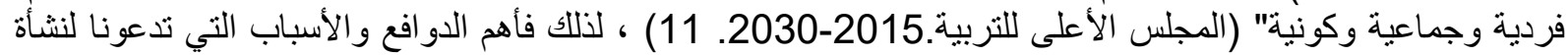

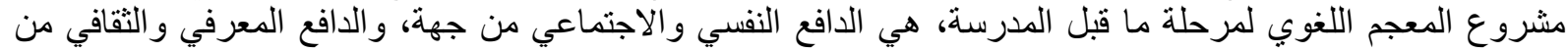
جهة ثنانية.
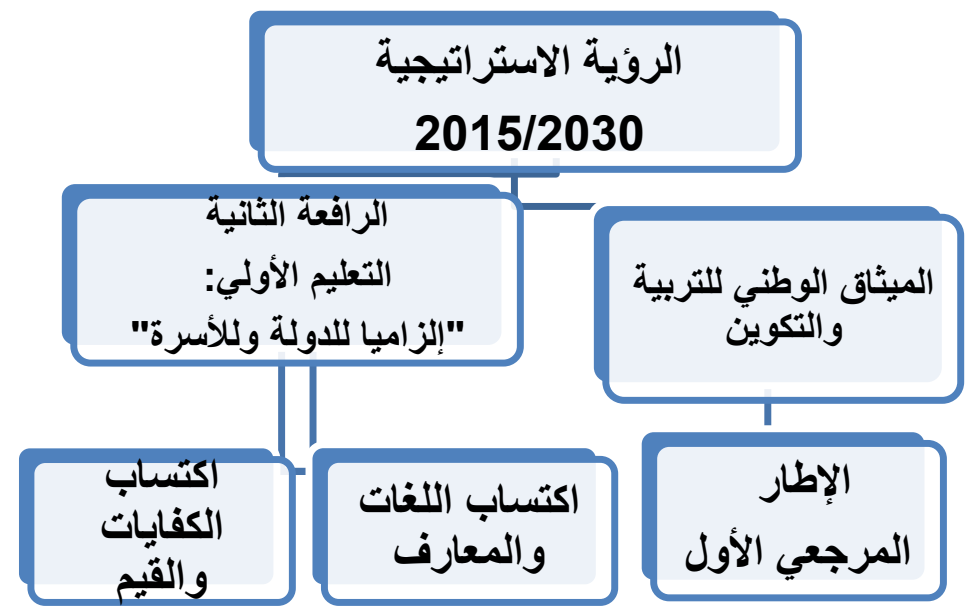

ب- - مفهوم الهندسة البيداغوجية خلال مرحلة التعليم الأولي:

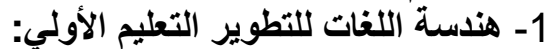

استثمار المكتسبات اللغوية و الثقافية الأولية للطفل و إدراج:

اللغة العربية

اللغة الفرنسية

التركيز على التواصل الثفهي انسجاما مع طبيعة هذا المستوى من التعليم.(المجلس الأعلى للتربية،2015-

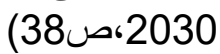

اعتبرت الرؤية التي تقدم بها المجلس الأعلى للتربية والتكوين و البحث العلمي، اللغة العربية هي لغة التدريس الأساس في

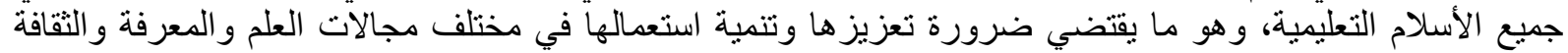




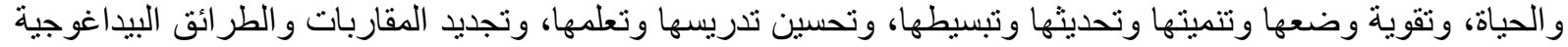

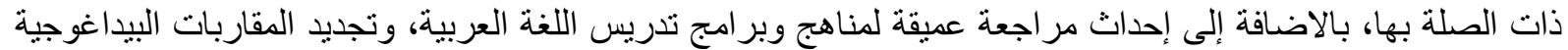

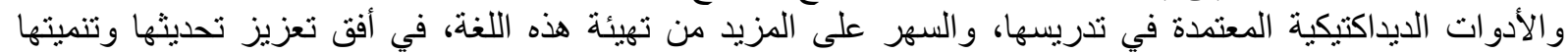

تـ الهناسة اللغوية الجديدة للمدرسة المغربية

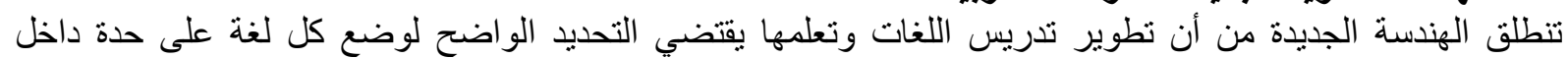

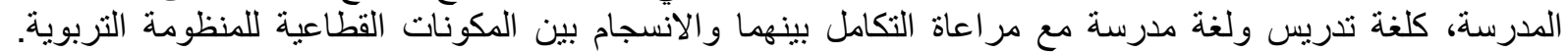

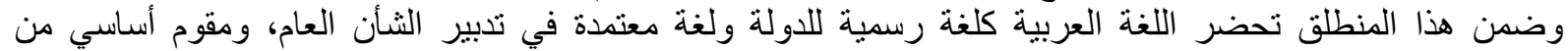

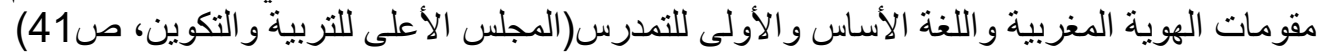

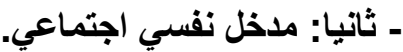

تتميز الحياة الاجتماعية المحيطة بالطفل خلاعل مرحلته العمرية الممتدة ما بين 4 إلى6 سنوات بكونها تحوّل ذكاء الطفل بواسطة الآليات اللغوية الثناثية الآتية:

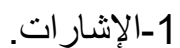

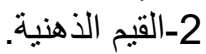
3-القيم الجماعية المنطقية.

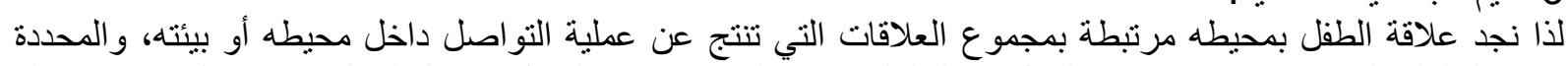

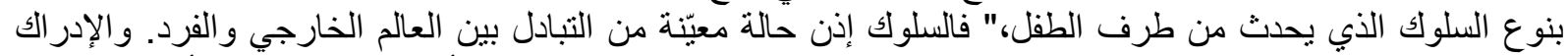

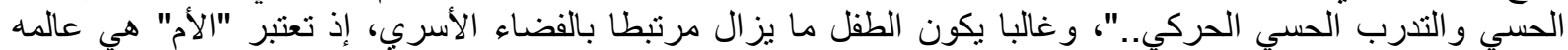
الاجتماعي بالأساس داخل الأسرة خلال تلألك المرحلة.

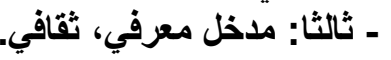

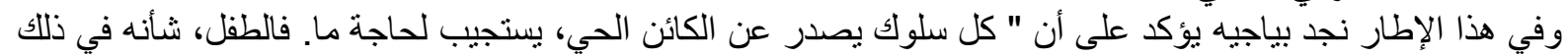

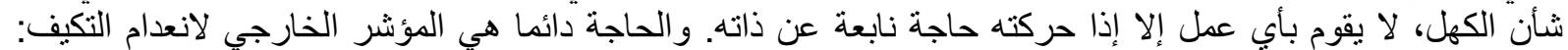

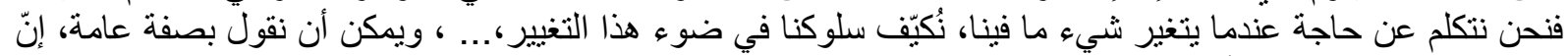

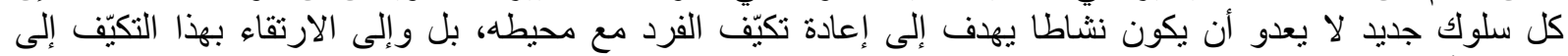

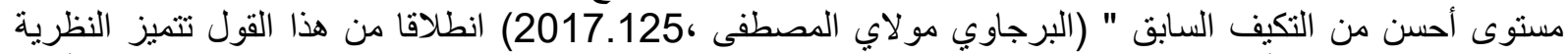

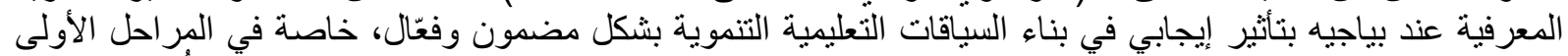

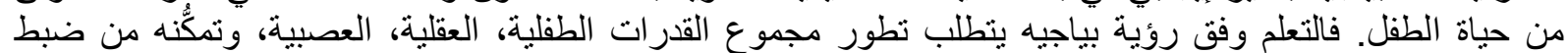

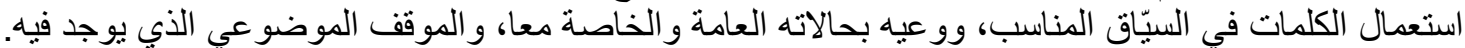

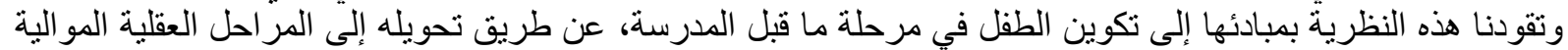
في عملية النمو، وذللك باحتو ائها على أربع مجالات رئيسة، تعد من التطبيقات التربوية الأساسية في العملية التعليمية

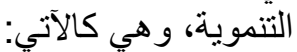

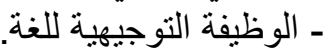

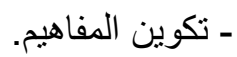

- ترجمة الخبر ات الحسية إلى أشكال رمزية. ـ ت تنمية التفكير المنطقي عند الأطفال.

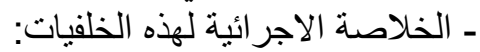




\section{PEDAGOGICAL ENGINEERING FOR TEACHING ARABIC FOR EARLY}

\section{CHILDHOOD-THE LEXICON AS A MODEL-}

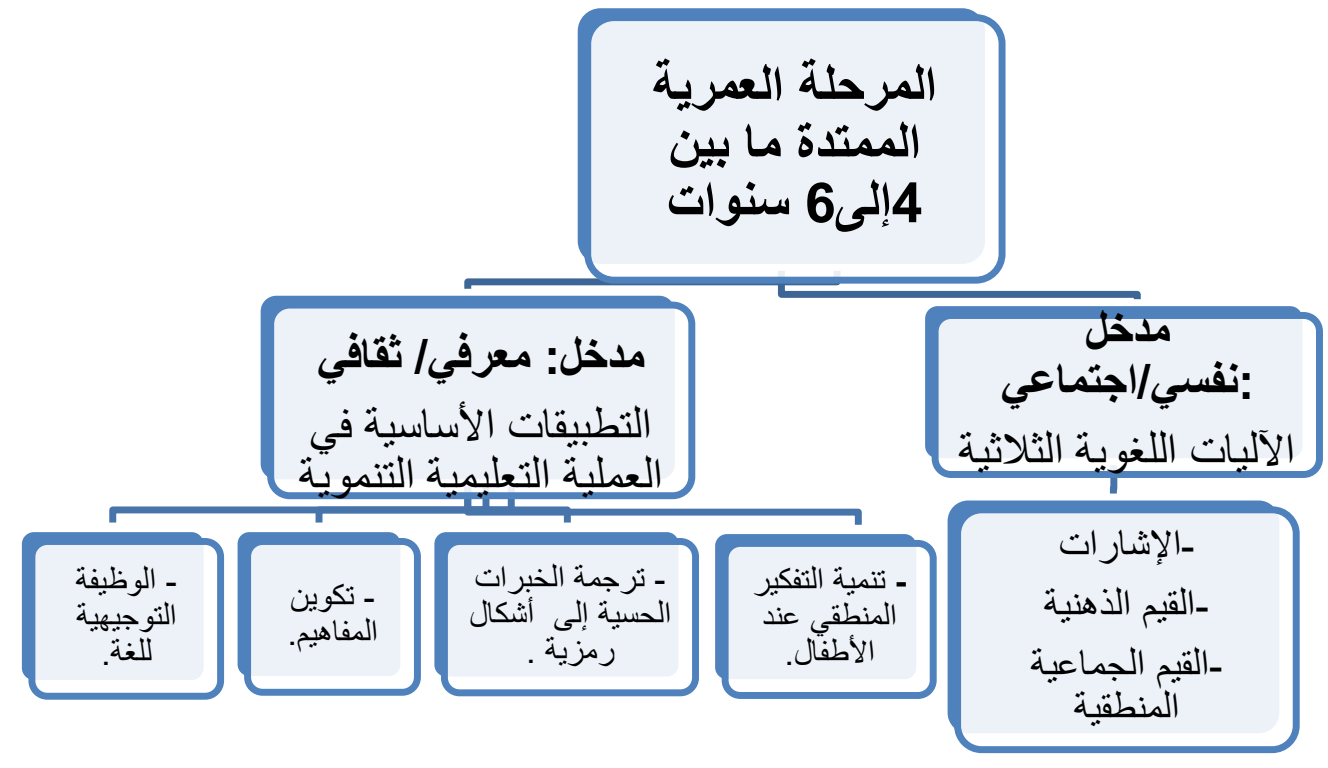

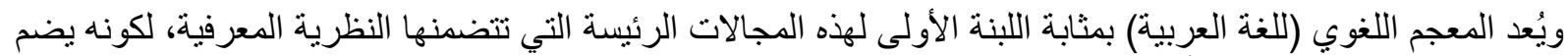

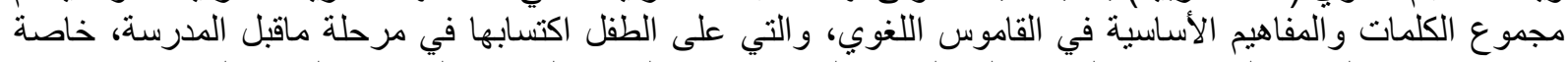

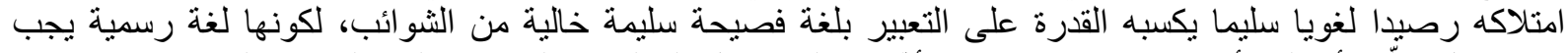

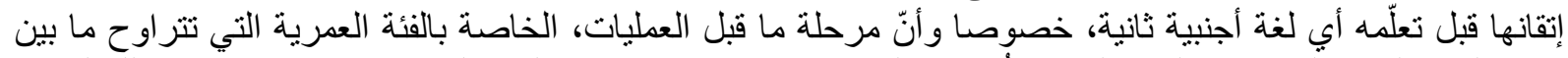

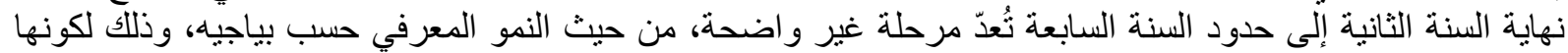

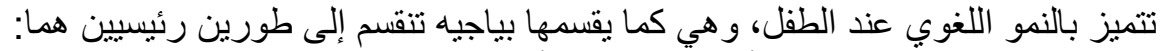

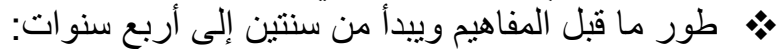

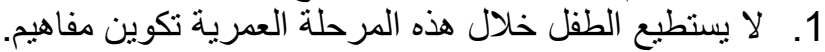

2. لا يستطيع الطفل خلال هذه المرحلة استعمال عملية التخييل أو الخيال.

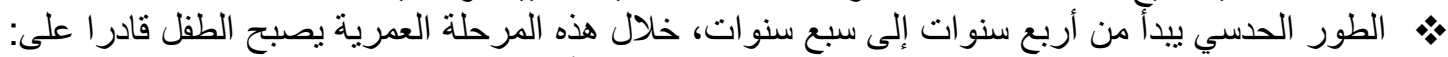

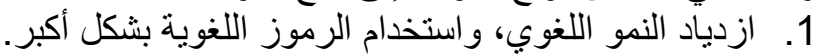

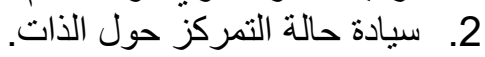
3. البدء بتكوين المفاهيم، وتصنيف النيف الأشياء.

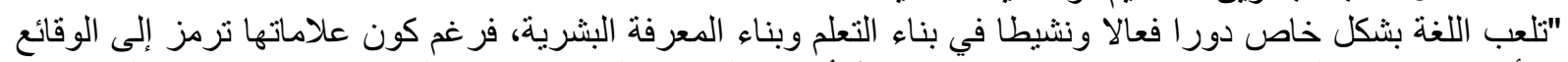

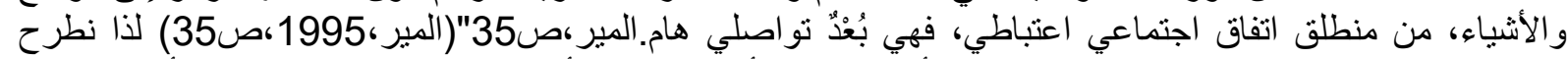

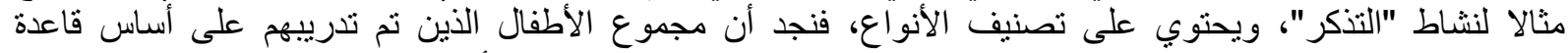

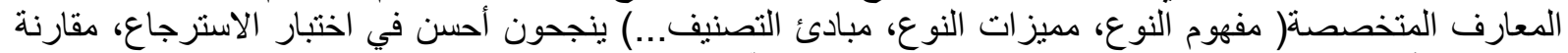

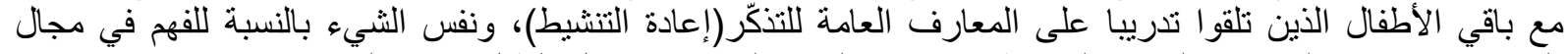

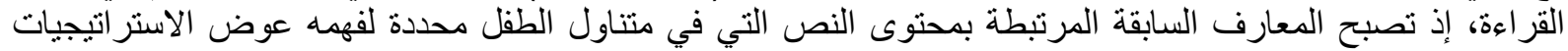
العامة للقر اءة.

\section{والخلاصة العملية الإجرائية هي:}

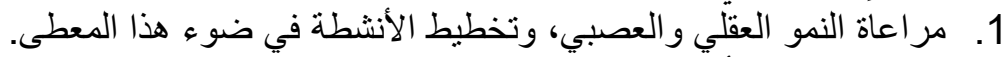

2.

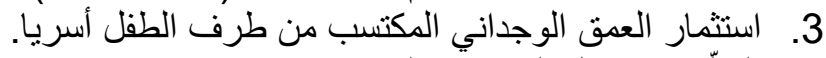

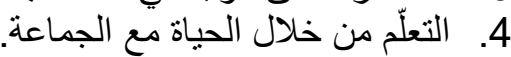

رابعا: مدخل الكفايات، والاختيار، والقيم، وبيان الخانيان الخلفية المتحكمة في المعجم في علاقة ذلكك بالروئية المنهجية للكفايات.

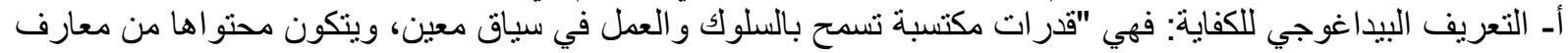

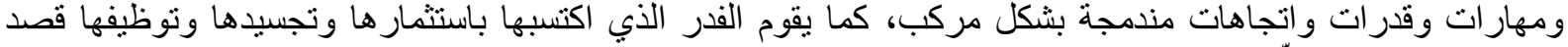

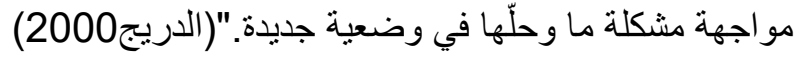




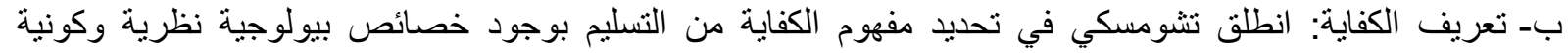
مسؤولة عن ذللك الاستعداد المسبق لدى كل فرد لاكتساب اللغة، فهي معارف ضمنية الفتراضية،ور طاقة فردية كامنة في

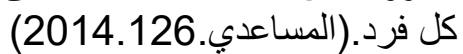

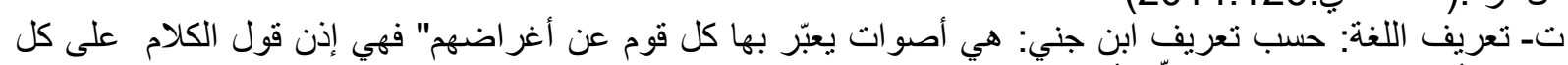

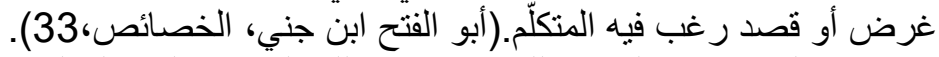

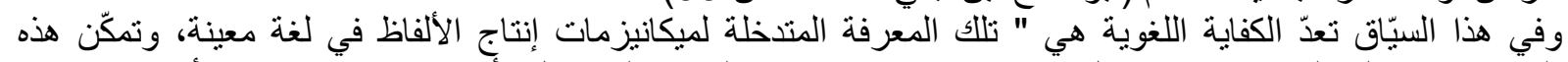

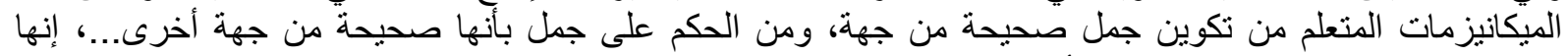

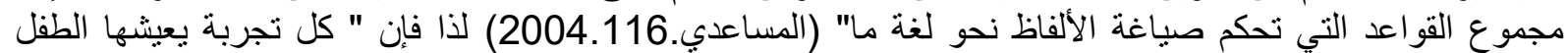

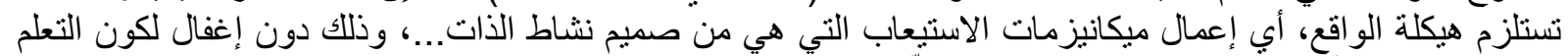

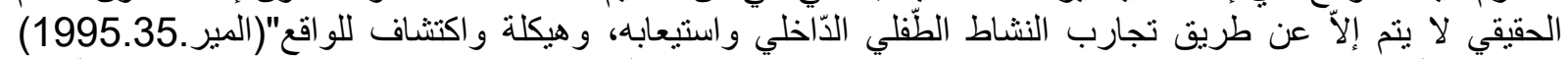

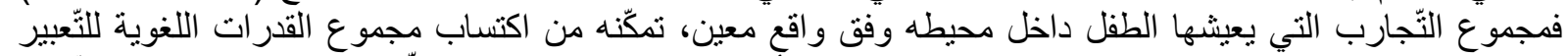

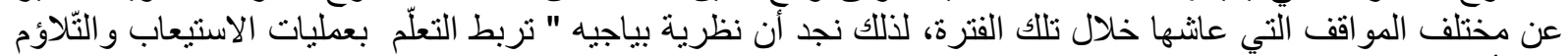

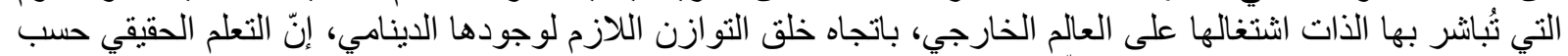

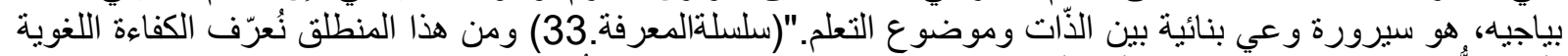

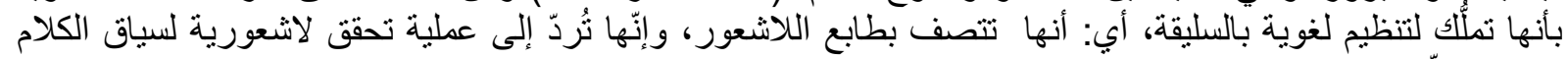

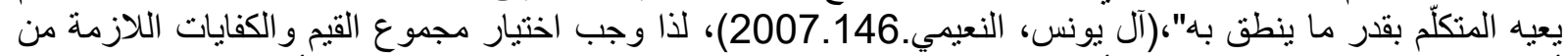

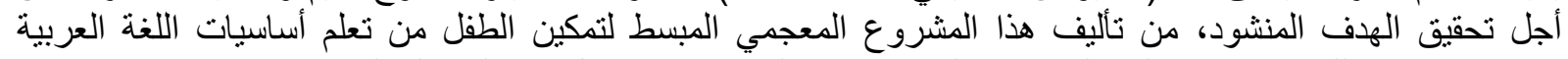

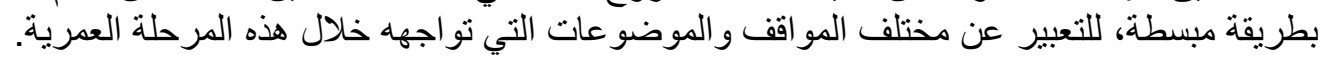
فعلى المعجم أن يحقق مايلي:

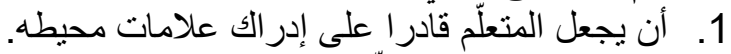

2. أن يجعل المعجم المتعلّم (الطفل) قادرا على إستعمال علامات على محيطه اللغوية (اللغة العربية)...استعمالا صحيحا مفيدا. 3. أن يساهم المعجم في بناء الكفايات لدى المتعلّم، ويكون قادرا من خلالها التعبير عن حاجاته.

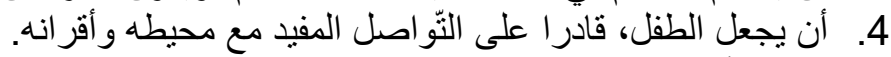

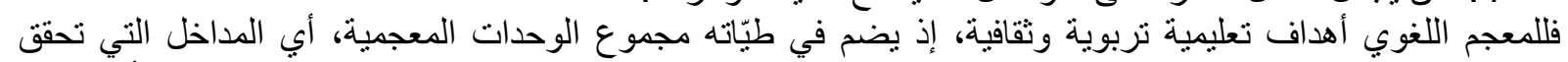

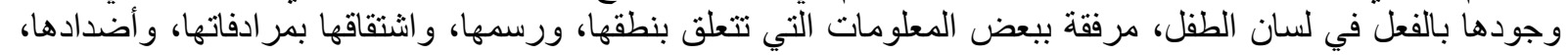

و واستعمالاتها، وتوظيفها.

أهمية المعجم: أهمية المعم اللغوي(العربي) الخاص بمرحلة التعليم ماقبل المدرسي فيما يلي:

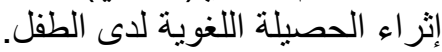
بناء قاعدة من المهار ات اللغوية لإى الطية الأولية (التحويل /التصريف/التركيب).

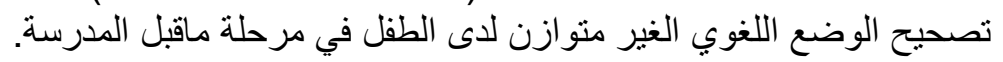

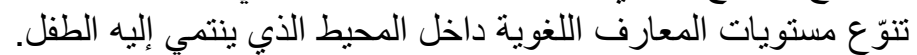

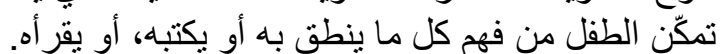

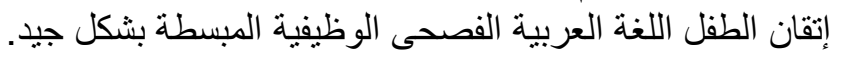

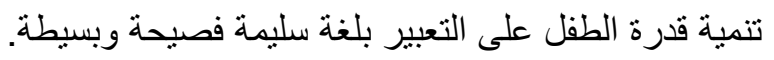

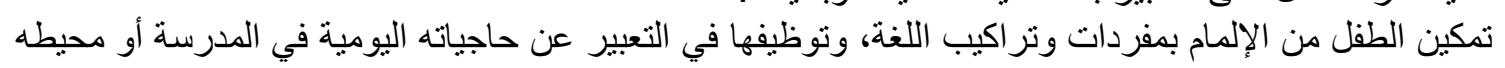
الاجتماعي: نطقا وكتابة.

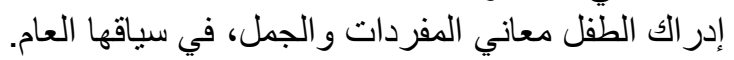

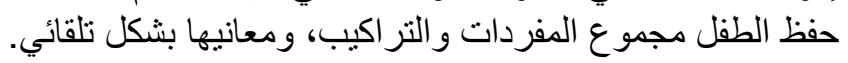

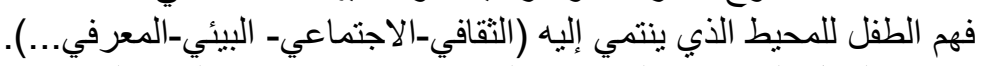

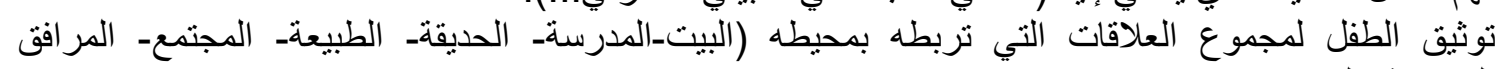
العمومية..إلخ). الرفع من مستوى الخطاب الفكري واللغوي اللغوي عند الطفل خلال مرحلة ماقبل المدرسة.

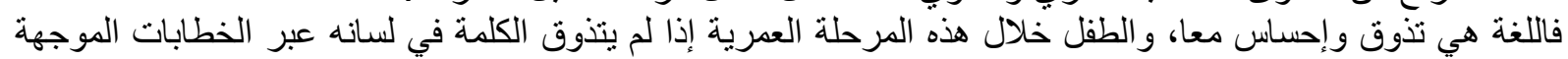

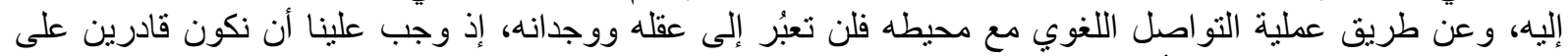

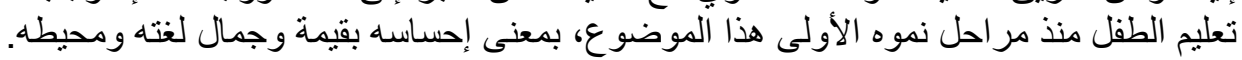




\section{PEDAGOGICAL ENGINEERING FOR TEACHING ARABIC FOR EARLY}

CHILDHOOD-THE LEXICON AS A MODEL-

المبحث الثاني: الخلقية الدياكتيكية المتحكمة في صناعة هذا المعجم. أولا: المبادئ الأساسية.

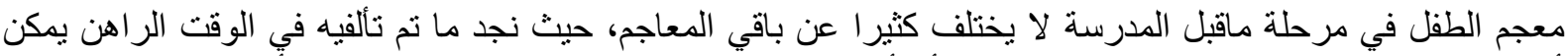

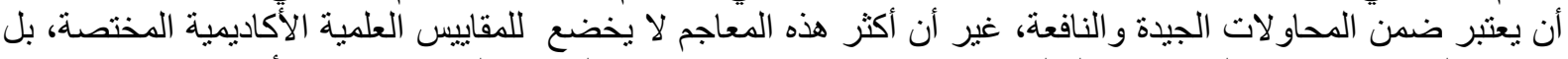

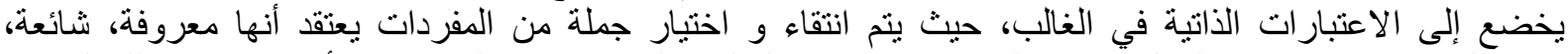

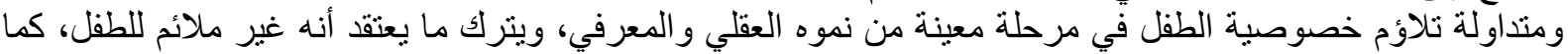

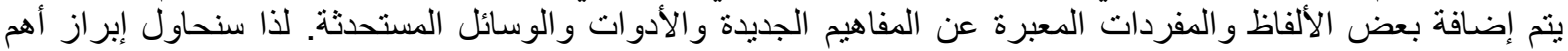

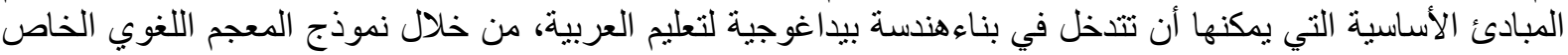
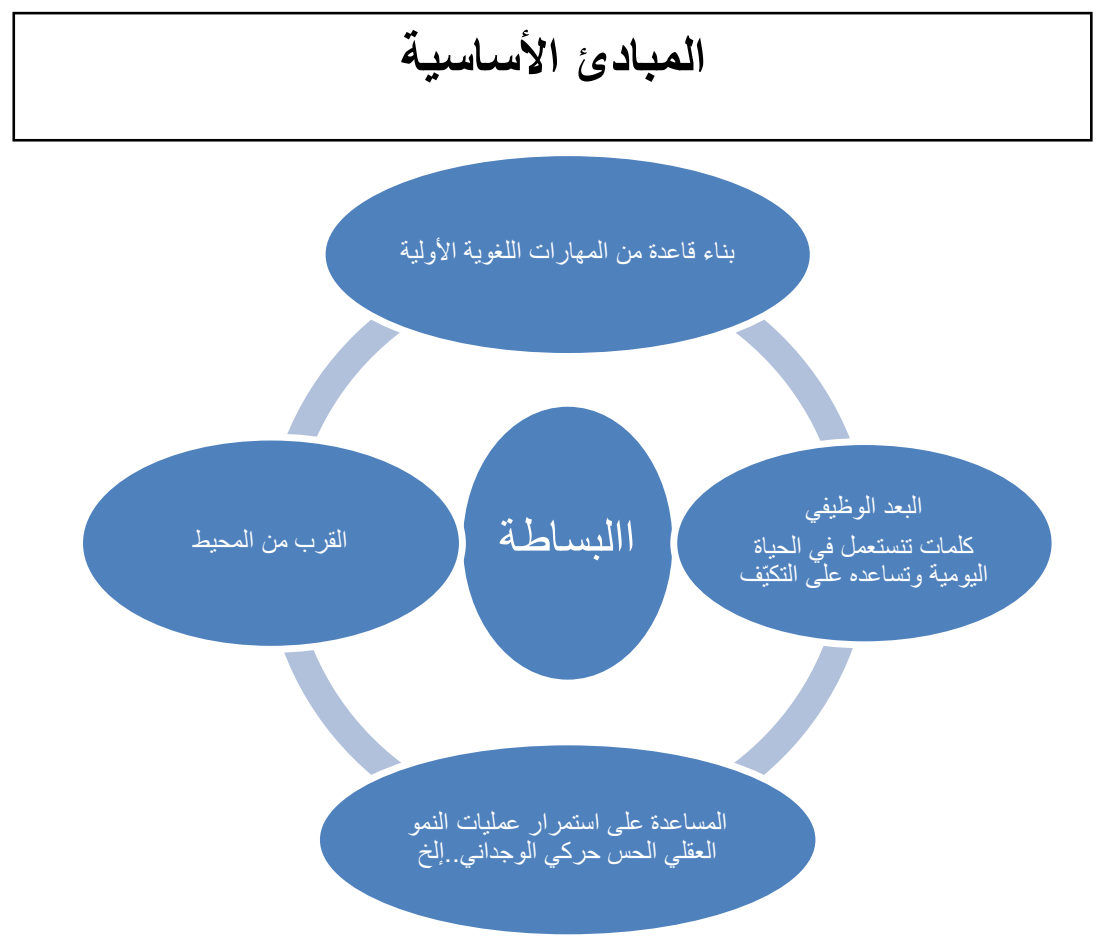

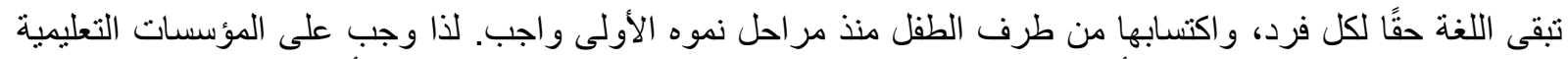

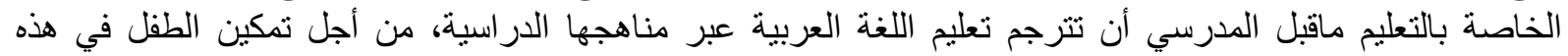

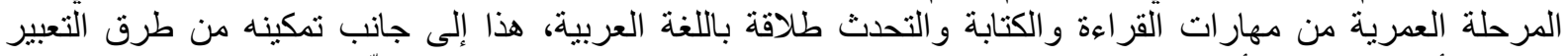

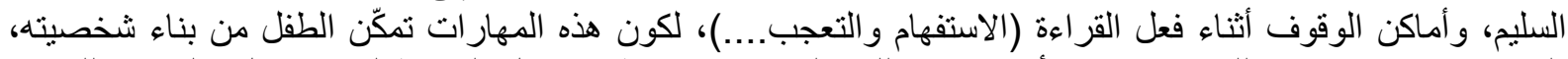

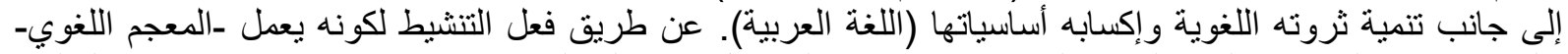

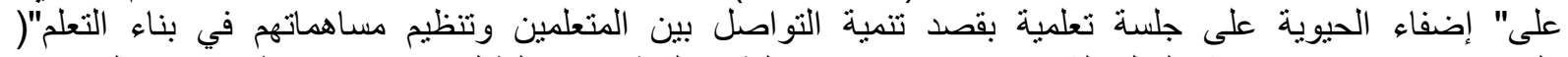

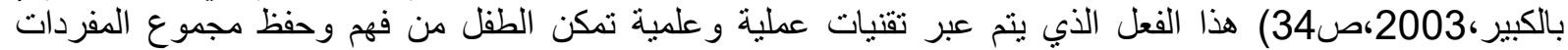
و التر اكيب بشكل تلقائي.

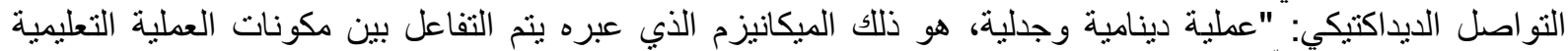

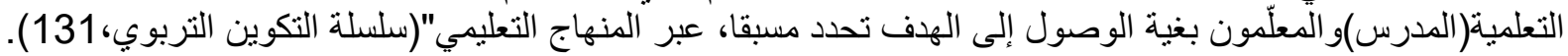

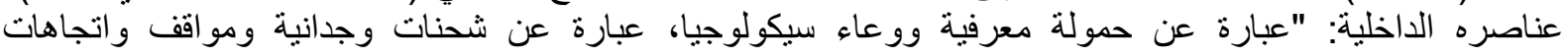
و إحساسات وكفاءات جسمية حركية. 


$$
\begin{aligned}
& \text { الخلاصة الإجر ائية تكمن في كون طفل ماقبل المدرسة علينا مساعدنه على: } \\
& \text { 1. التعلم عن طريق اللعب (حس حركي). } \\
& \text { 2. التعلم عن طريق التنشيط(التو اصل بلئ بين المربي و الطفل). } \\
& \text { 3. البعد الحسي (مادي وليس مجرد). } \\
& \text { 4. التعلم عن طريق الموسيقى. }
\end{aligned}
$$

يصنف المتخصصون في علم المثري المفرد ألفاظ اللغة إلى ثُلاث مستويات:

1. ما يعرفه المتكلم و ولا بستطيع استعماله.

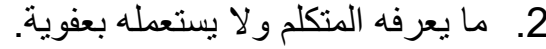

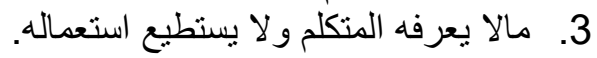

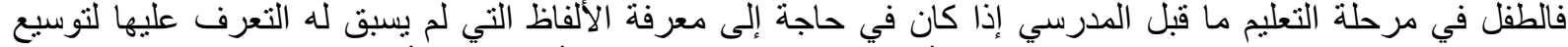

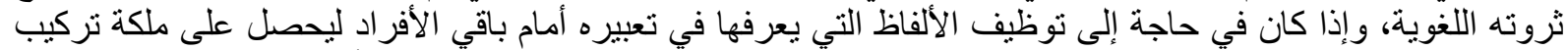

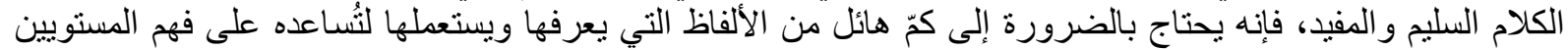

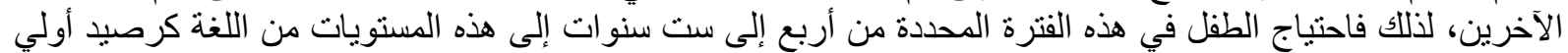

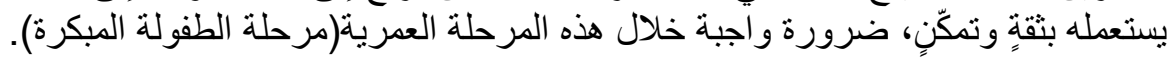

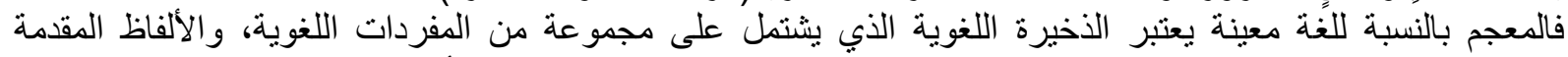

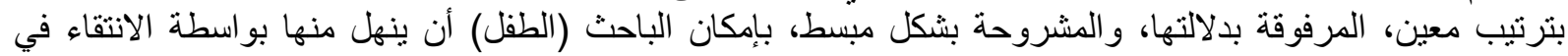

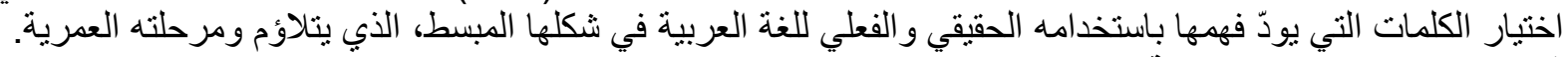

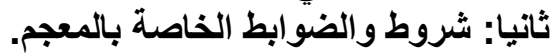

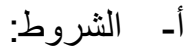

$$
\begin{aligned}
& \text { • • وضع مصطلحات علمية وفنّية معاصرة. }
\end{aligned}
$$

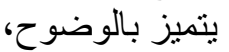

$$
\begin{aligned}
& \text { يتميز بالثمولية لكل مايتعلّق بمرحلة الطفولة المبكرة }
\end{aligned}
$$

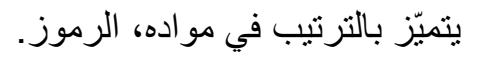

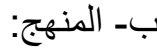

$$
\begin{aligned}
& \text { وصفي تحليلي في ترتيب مو اده، مفرداته، وأساليب، وجمله. }
\end{aligned}
$$

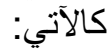

$$
\begin{aligned}
& \text { تقديم الأفعال على الأسماء. } \\
& \text { تقديم الأفعال المجردة على الألى المزيدة. } \\
& \text { فصل الأفعال المتعدية على الأفعال اللازمة. }
\end{aligned}
$$

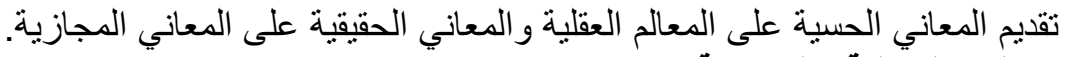

$$
\begin{aligned}
& \text { ثالثا: المواصفات الداخلية والخارجية } \\
& \text { أ- على مستوى الثكل: }
\end{aligned}
$$

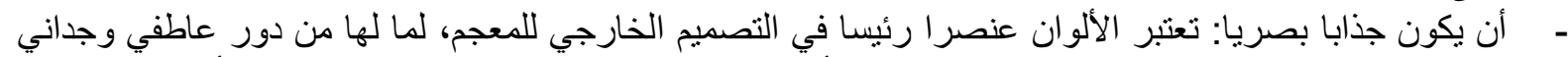

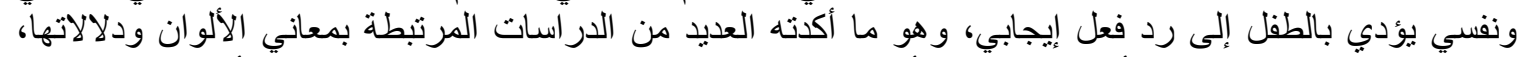

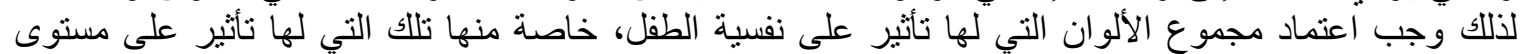

اعتماد اللون الأصفر لما: له من تأثثر إيجابي + يكثر نسبة التركيز عند الطفل+ يعمل على تنتيط

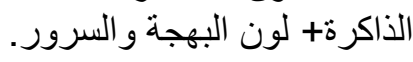

اللون البرتقالي: لون التحفيز والإبداع+ يجعل الطفل يبتكر ويبدع+ لون محبب إلى اللى النفس، اجتماعي.

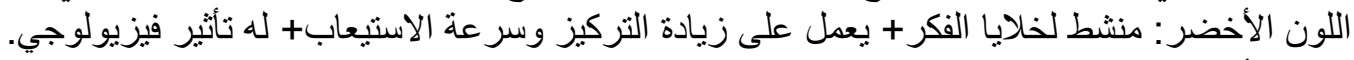

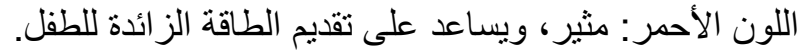

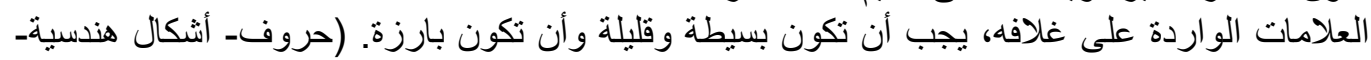




$$
\text { - - أن يساعده في بناء المهار ات الأساسية التي يحتاجها (التحويل ـ التكملة ـ التركيب - التمبيز ...). }
$$

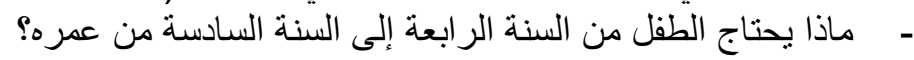

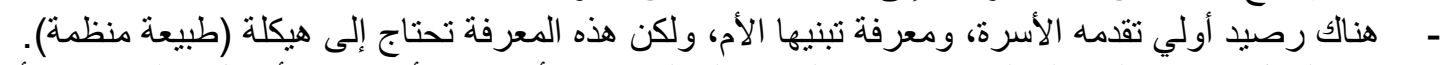

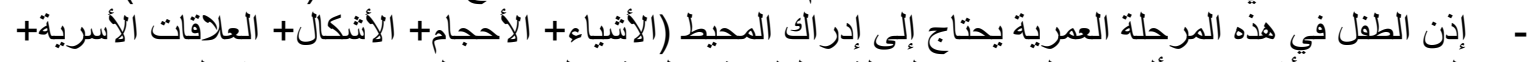

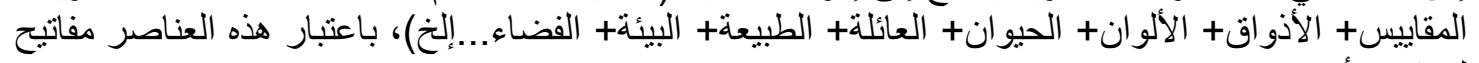

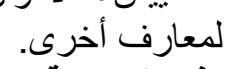
رابعا: نموذج الوضعية

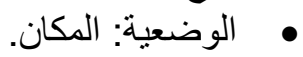

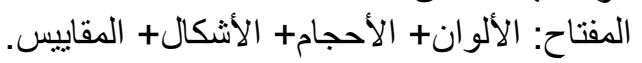

القيمة: تقدير أهمية المحيط.

$$
\checkmark
$$

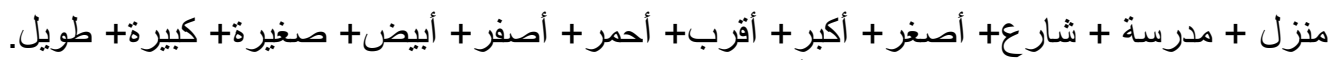

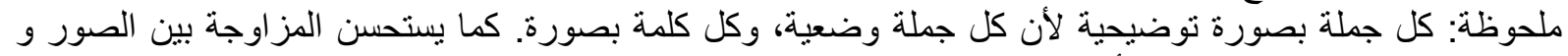
اللوحات،( على الصور واللوحات أن تكون من صميم الحياة الاجتماعية للطفل ) .

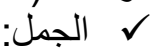

$$
\begin{aligned}
& \text { • منزل كبير. } \\
& \text { • مدرسة صغيرة. }
\end{aligned}
$$

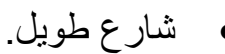

$$
\begin{aligned}
& \text { ل } \\
& \text { جدار المنزل أصفر. } \\
& \text { علم المدرسة أحمر. }
\end{aligned}
$$

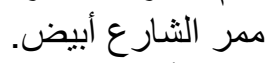

$$
\begin{aligned}
& \text { منزلنا أكبر منزل في الحي. } \\
& \text { • • • مدرستي أقرب مكان لي. } \\
& \text { • • في الثار ع الر ابع أصغر عمارة. } \\
& \text { الفوائد المحققة: } \\
& \text { تموقع الطفل في المكان. } \\
& \text { تقدير الطفل قيمة المكان. } \\
& \text { • قدرة الطفل على وصف المكان. } \\
& \text { • • • • • • • }
\end{aligned}
$$

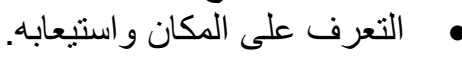

$$
\begin{aligned}
& \text { حفظ الطفل اسم المكان. } \\
& \text { • • قدرة الطفل على تحديد المكان. } \\
& \text { • • • • •مكين الطفل من مر ادفات المكان. } \\
& \text { • • معرفة الطفل معاني المكان. }
\end{aligned}
$$

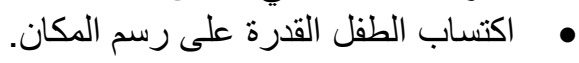

$$
\begin{aligned}
& \text { • • • • استمتاع الطفل بجمال المكان. }
\end{aligned}
$$




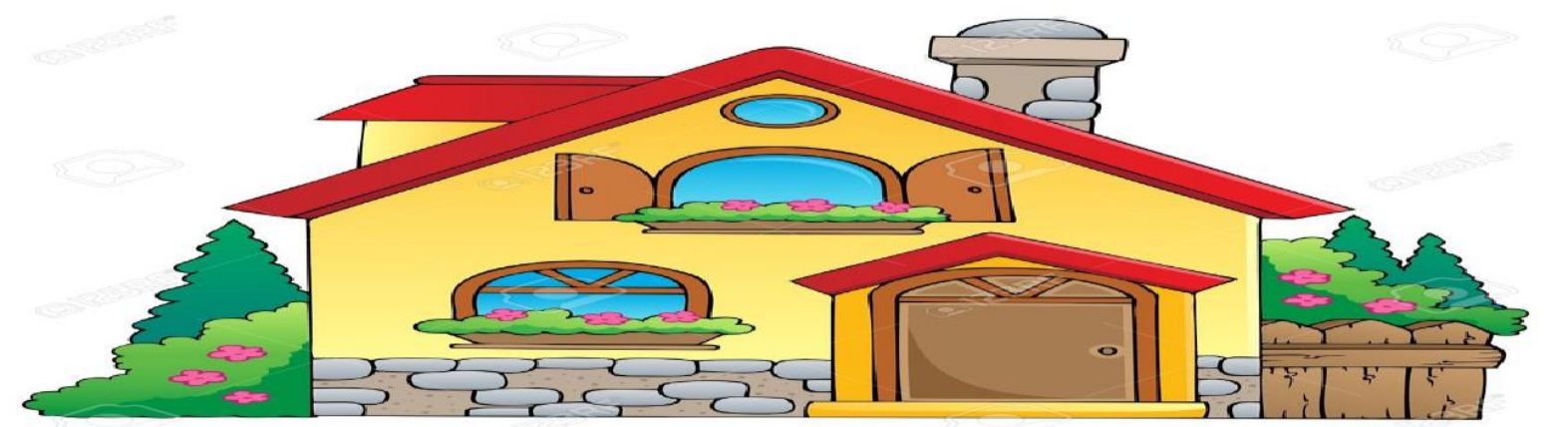

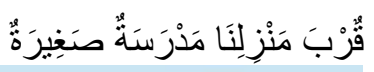

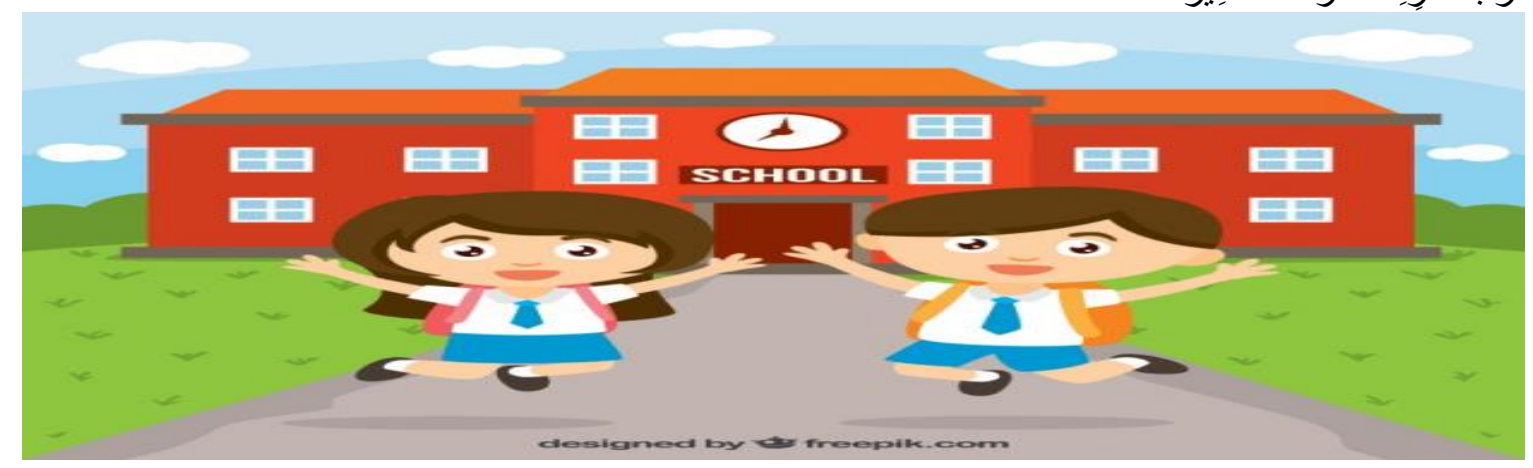

أعْْرُ شَدَرِ عَاً طَوِلًا

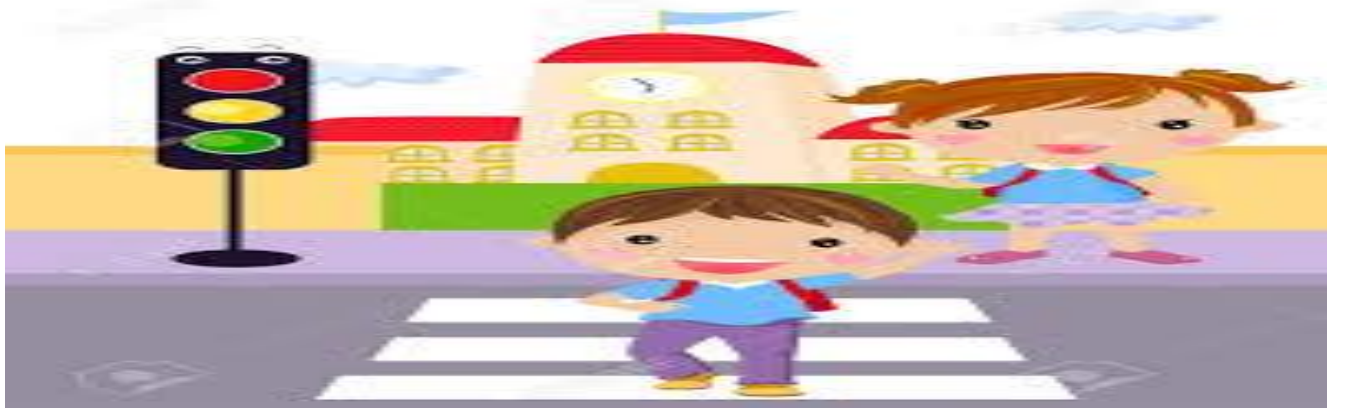

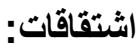

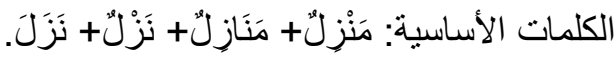

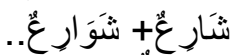

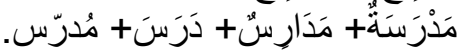

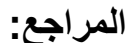

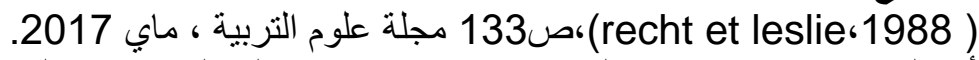

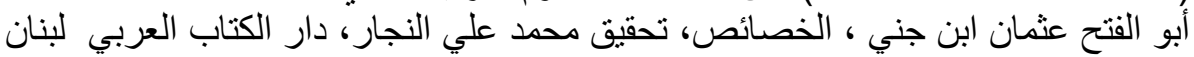

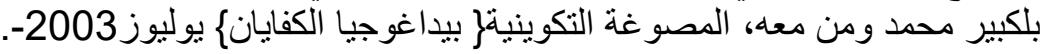

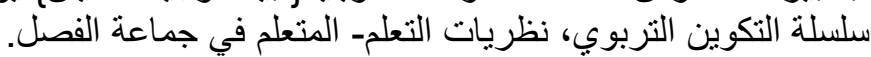

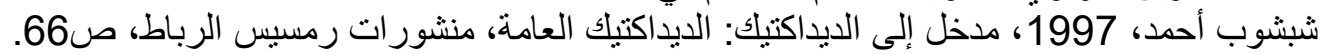

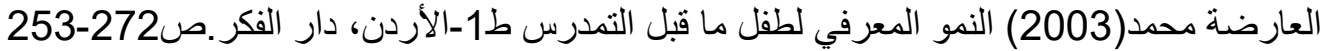


علي عون عيشة علة- نظير بياجي للتنمية المعرفية الآليات التنموية و التداعيات التعليمية، مجلد 2، عدد2، جوان2019.

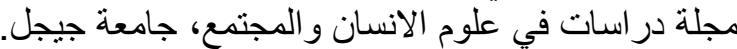

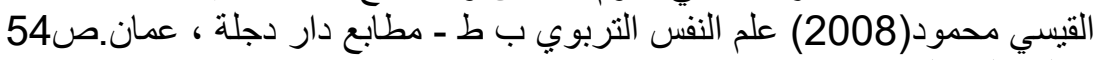

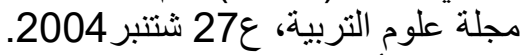

المجلس الأعلى للتربية و التكوين و البحث العلمي، من أجل مدرسة الإنصاف الجودة والارتقاء، رؤية إستر اتيجية للإصلاح 2030-2015

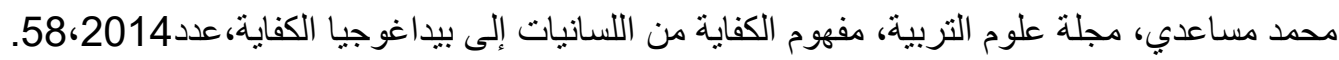

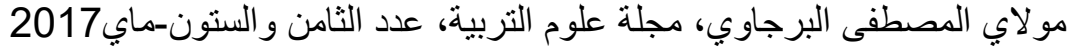

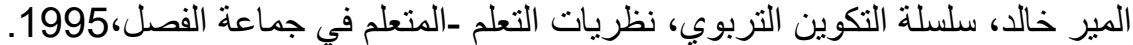

هاني صبري آل يونس، وسلوى خضر فتحي، النعيمي، مجلة التربية والعلم، الكفاءة اللغوية وتعيين

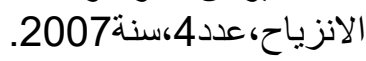

